\title{
Healthcare professionals' and dieters' attitudes and perceptions of obesity
}

\author{
Á. McConnon ${ }^{1}$, M. M. Raats ${ }^{1}$, R. Shepherd ${ }^{1}$ and J. Stubbs ${ }^{2}$ \\ ${ }^{1}$ Food, Consumer Behaviour and Health Research Centre, Faculty of Arts and Human Sciences, University of Surrey, \\ Guildford, Surrey GU2 7XH, UK and ${ }^{2}$ Nutrition Department, Slimming World, Alfreton, Derbyshire DE55 4RF, UK
}

Health professionals have a major role to play in addressing obesity and overweight in the general population ${ }^{1}$. Differences in beliefs and attitudes to obesity between healthcare providers and individuals attempting weight control are likely to act as a barrier in successful weight management. The aim of the present research was to investigate differences in attitudes and perceptions of obesity between healthcare providers and overweight and obese individuals.

A web-based questionnaire designed to measure attitudes, perceptions, beliefs and barriers to successful weight control was developed. Healthcare participants were recruited through mailing lists of professional organisations for health professionals and students with an interest in obesity. Dieters were recruited via email advertisement sent out to companies in the Surrey Research Park, Guildford, UK. A five-point scale from 1 (not at all) to 5 (totally) was used, mean scores were produced.

The questionnaire was completed by 283 health professionals and 116 dieters. The majority of the sample was female (82\%) and married or living with a partner $(70 \%)$. The data on beliefs about the causes of obesity were factor analysed and four factors were produced (see Table). Both groups believe that 'lifestyle factors' have the greatest role to play in causing obesity. These factors include unhealthy food choices, lack of exercise, food portion size and lack of self control.

\begin{tabular}{|c|c|c|c|c|}
\hline & Mean scores & Mean difference & $P$ & $95 \% \mathrm{CI}$ \\
\hline \multirow[t]{2}{*}{ Lifestyle factors } & HP: 3.9 & & & \\
\hline & Dieters: 3.8 & 0.16 & 0.8 & $-0.1,0.1$ \\
\hline \multirow[t]{2}{*}{ Medical factors } & HP: 2.0 & & & \\
\hline & Dieters: 2.5 & 0.54 & $<0.001$ & $0.4,0.7$ \\
\hline \multirow[t]{2}{*}{ Psychological factors } & HP: 3.0 & & & \\
\hline & Dieters: 2.6 & -0.4 & $<0.001$ & $-0.6,-0.2$ \\
\hline \multirow[t]{2}{*}{ Social factors } & HP: 3.2 & & & \\
\hline & Dieters: 3.2 & 0.01 & 0.95 & $-0.19,0.2$ \\
\hline
\end{tabular}

Significant differences were revealed between the groups for 'medical factors' and 'psychological factors', with health professionals more likely than dieters to view psychological factors as important and dieters more likely to view 'medical factors' as an important cause of obesity. There was a significant difference $(P \leq 0.001)$ in beliefs about the effects of obesity, with health professionals reporting factors such as driving, going to the toilet, dressing, basic interpersonal interaction, family relationships, educational attainment and fertility, affect obesity more than dieters think they do. Differences in attitudes to a range of methods for dealing with obesity were revealed between the two samples. Health professionals were significantly more positive about surgery (mean score; health professionals 3.19 , dieters $2.68 ; P<0.001$ ) and visiting a psychologist (mean score; health professionals 3.03 , dieters $2.63 ; P=0.001$ ). However, dieters were more positive about yoga (mean score; health professionals 2.69 , dieters $2.33 ; P<0.001$ ) and 'making big changes to one's lifestyle' (mean score; health professionals 3.57 , dieters $3.91 ; P=0.006$ ).

Differences in attitudes and beliefs about obesity held by health professionals and individuals attempting weight loss should be addressed in order to inform successful weight-control strategies.

1. National Institutes of Health, National Heart Lung and Blood Institute (1998) Clinical Guidelines on the Identification, Evaluation, and Treatment of Overweight and Obesity in Adults. Bethesda, MD: National Institutes of Health. 